\title{
Operator properties of generalized coherent state systems
}

\author{
N MUKUNDA* \\ Centre for Theoretical Studies and Department of Physics, Indian Institute of Science, \\ Bangalore 560 012, India \\ ${ }^{*}$ Honorary Professor, Jawaharlal Nehru Centre for Advanced Scientific Research, Jakkur, \\ Bangalore 560 064, India
}

\begin{abstract}
The main properties of standard quantum mechanical coherent states and the two generalizations of Klauder and of Perelomov are reviewed. For a system of generalized coherent states in the latter sense, necessary and sufficient conditions for existence of a diagonal coherent stable representation for all Hilbert-Schmidt operators are obtained. The main ingredients are Clebsch-Gordan theory and induced representation theory.
\end{abstract}

Keywords. Generalized coherent states; diagonal representation; induced representation theory.

PACS Nos 03.65.-w; 03.65.Ca; 02.20.Qs

\section{Introduction}

The aim of these two lectures is to review very briefly the properties of the standard coherent states in canonical quantum mechanics; sketch the main features of the two generalisations of the coherent state concept due respectively to Klauder [1] and to Perelomov [2]; and against this background to discuss the question of descriptions of operators in terms of a given generalized coherent state (GCS) system. Specifically our target is the derivation of necessary and sufficient conditions that ensure that any operator can be given a diagonal coherent state representation, namely expressed as an integral over projections onto the set of generalised coherent states. We shall in fact derive such conditions within the Perelomov framework, and the principal tool we use is the reciprocity theorem concerning the reduction of induced group representations into irreducibles.

Various definitions and terms will be clarified as we proceed.

\section{The standard coherent states - a review [3]}

Let us limit ourselves for simplicity to a quantum mechanical system built upon a single canonical pair of operators. Thus we have a hermitian pair of operators $\hat{q}, \hat{p}$ or equivalently the annihilation and creation operator pair $\hat{a}, \hat{a}^{\dagger}$. We first recall the basic operator aspects and relations, then turn to the coherent states.

The canonical Heisenberg commutation relations, written in either form, are: 


$$
\begin{gathered}
{[\hat{q}, \hat{p}]=i ;} \\
\hat{a}=\frac{1}{\sqrt{2}(\hat{q}+i \hat{p})}, \hat{a}^{\dagger}=\frac{1}{\sqrt{2}}(\hat{q}-i \hat{p}), \\
{\left[\hat{a}, \hat{a}^{\dagger}\right]=1 .}
\end{gathered}
$$

By the Stone-von Neumann theorem, up to unitary equivalence there is just one irreducible representation of these relations [4]. The Hilbert space $\mathcal{H}$ of this representation can be realised as the space $\ell^{2}$ or the space $L^{2}(\mathcal{R})$ of square integrable functions over the real line. Correspondingly we have a Fock basis for $\mathcal{H}$, or the Schrödinger basis made up of (ideal) eigenvectors of the position operator $\hat{q}$ :

$$
\begin{aligned}
\hat{a}^{\dagger} \hat{a}|n\rangle & =n|n\rangle, n=0,1,2, \ldots, \infty \\
\langle m \mid n\rangle & =\delta_{m n} \\
\hat{q}|q\rangle & =q|q\rangle,-\infty<q<\infty \\
\left\langle q^{\prime} \mid q\right\rangle & =\delta\left(q^{\prime}-q\right)
\end{aligned}
$$

A general vector $|\psi\rangle \in \mathcal{H}$ is describable either by its expansion coefficients in the discrete basis $|n\rangle$, or by its Schrödinger wave function $\psi(q)$ :

$$
\begin{aligned}
\langle n \mid \psi\rangle & =\psi_{n} \\
\langle q \mid \psi\rangle & =\psi(q), \\
\langle\psi \mid \psi\rangle=\||\psi\rangle \|^{2} & =\sum_{n \in \mathcal{Z}}\left|\psi_{n}\right|^{2}=\int_{-\infty}^{\infty} \mathrm{d} q|\psi(q)|^{2} .
\end{aligned}
$$

For any choice of a complex number $z=\frac{1}{\sqrt{2}}(q+i p)$, where $q$ and $p$ are possible (but of course not simultaneous) eigenvalues of $\hat{q}$ and $\hat{p}$ respectively, we define the unitary displacement operator $D(z)$ by

$$
\begin{aligned}
D(z) & \equiv D(q, p) \\
& =\exp \left(z \hat{a}^{\dagger}-z^{*} \hat{a}\right) \\
& =\exp \{i(p \hat{q}-q \hat{p})\} \\
D(z)^{\dagger} D(z) & =1 .
\end{aligned}
$$

Their composition law and actions on the basic canonical operators are:

$$
\begin{aligned}
D\left(z^{\prime}\right) D(z) & =\exp \left\{i \operatorname{Im} z^{\prime} z^{*}\right\} D\left(z^{\prime}+z\right) \\
& =\exp \left\{\frac{i}{2}\left(p^{\prime} q-q^{\prime} p\right)\right\} D\left(q^{\prime}+q, p^{\prime}+p\right) ; \\
D(z)^{-1}\left(\hat{q}, \hat{p}, \hat{a}, \hat{a}^{\dagger}\right) D(z) & =\hat{q}+q, \hat{p}+p, \hat{a}+z, \hat{a}^{\dagger}+z^{*} .
\end{aligned}
$$

The displacement operators form an (ideal) orthonormal basis for the space of Hilbert Schmidt operators on $\mathcal{H}$. We define this space $\mathcal{K}$, a 'second' Hilbert space, as made up of operators $\hat{A}, \hat{B}, \ldots$ on $\mathcal{H}$ with the inner product given as follows: 


$$
\begin{aligned}
\hat{A}, \hat{B} \in \mathcal{K}:(\hat{A}, \hat{B}) & =\operatorname{Tr}\left(\hat{A}^{\dagger} \hat{B}\right), \\
\|\hat{A}\|^{2} & =\operatorname{Tr}\left(\hat{A}^{\dagger} \hat{A}\right)
\end{aligned}
$$

With respect to this inner product in $\mathcal{K}$, we find:

$$
\begin{aligned}
\left(D\left(q^{\prime}, p^{\prime}\right), D(q, p)\right) & =\operatorname{Tr}\left(D\left(q^{\prime}, p^{\prime}\right)^{\dagger} D(q, p)\right) \\
& =2 \pi \delta\left(q^{\prime}-q\right) \delta\left(p^{\prime}-p\right)
\end{aligned}
$$

and for a general operator $\hat{A} \in \mathcal{K}$ we have an expansion in terms of the displacement operators:

$$
\begin{aligned}
\hat{A} \in \mathcal{K}: \hat{A} & =\iint \frac{\mathrm{d} q \mathrm{~d} p}{2 \pi} a(q, p) D(q, p), \\
a(q, p) & =(D(q, p), \hat{A}), \\
\|\hat{A}\|^{2} & =\iint \frac{\mathrm{d} q \mathrm{~d} p}{2 \pi}|a(q, p)|^{2} .
\end{aligned}
$$

The expansion coefficient $a(q, p)$ is the Weyl weight of $\hat{A}$.

Against this background we now recapitulate the definition and most important properties of the standard coherent states. For each complex number $z=\frac{1}{\sqrt{2}}(q+i p)$ we have one coherent state $|z\rangle$ :

$$
\begin{aligned}
|z\rangle & =D(z)|0\rangle \in \mathcal{H}, \\
\hat{a}|z\rangle & =z|z\rangle, \\
\hat{a}|0\rangle & =0,
\end{aligned}
$$

where $|0\rangle$ is the Fock state $|0\rangle$ for $n=0$. Then we find:

(i) These states are never mutually orthogonal:

$$
\left\langle z^{\prime} \mid z\right\rangle=\exp \left\{-i \operatorname{Im} z^{\prime} z^{*}-\frac{1}{2}\left|z^{\prime}-z\right|^{2}\right\} \neq 0 .
$$

(ii) Their Schrödinger wave functions are Gaussian:

$$
\left\langle q^{\prime} \mid z\right\rangle=\pi^{-1 / 4} \exp \left\{i p\left(q^{\prime}-q / 2\right)-\frac{1}{2}\left(q^{\prime}-q\right)^{2}\right\} .
$$

(iii) The uncertainties $\Delta q, \Delta p$ are equal, the uncertainty principle is saturated and there is no squeezing, in any $|z\rangle$ :

$$
\Delta q=\Delta p=\frac{1}{\sqrt{2}}
$$


(iv) There is a resolution of the identity:

$$
\begin{aligned}
\int \frac{\mathrm{d}^{2} z}{\pi}|z\rangle\langle z| & =1 \\
|\psi\rangle \in \mathcal{H}:|\psi\rangle & =\int \frac{\mathrm{d}^{2} z}{\pi}|z\rangle\langle z \mid \psi\rangle .
\end{aligned}
$$

(v) This association of a 'wave function' $\langle z \mid \psi\rangle$ with each $|\psi\rangle \in \mathcal{H}$ leads to the Bargmann description of $\mathcal{H}$ using entire functions $f(z)$ of a certain class:

$$
\begin{aligned}
\left\langle z^{*} \mid \psi\right\rangle & =\exp \left(-\frac{1}{2}|z|^{2}\right) \sum_{n=0}^{\infty} \psi_{n} z^{n} / \sqrt{n !} \\
& =\exp \left(-\frac{1}{2}|z|^{2}\right) f(z) \\
f(z) & =\sum_{n=0}^{\infty} \psi_{n} z^{n} / \sqrt{n !}=\text { entire analytic; } \\
|f(z)| & \leq \exp \left(\frac{1}{2}|z|^{2}\right)\|\psi\| ; \\
\|\psi\|^{2} & =\int \frac{\mathrm{d}^{2} z}{\pi} \mathrm{e}^{-|z|^{2}}|f(z)|^{2}
\end{aligned}
$$

In this description, specially suited to the actions of $\hat{a}$ and $\hat{a}^{\dagger}$ on $|\psi\rangle$, we have

$$
\hat{a} \rightarrow \frac{\mathrm{d}}{\mathrm{d} z}, \hat{a}^{\dagger} \rightarrow z
$$

(vi) There exist characteristic sets $S \subset C$, subsets of the complex plane, such that

$$
f(z)=0, \text { all } z \in S \Rightarrow|\psi\rangle=0 .
$$

Examples are: any discrete infinite sequence with a finite limit point; any open continuous interval of the real axis, or of the imaginary axis; any finite continuous arc in the complex plane, any bounded open subset of the complex plane, etc. [5]

(vii) Properties (i) and (iv) above show that the coherent states form an overcomplete family. As a result we find that the diagonal coherent state matrix elements $\langle z|\hat{A}| z\rangle$ of a general operator $\hat{A}$ on $\mathcal{H}$ determine $\hat{A}$ completely.

(viii) An even more striking consequence of over completeness is the following: any operator $\hat{A}$ has a (unique) diagonal representation

$$
\hat{A}=\int \frac{\mathrm{d}^{2} z}{\pi} \phi(z)|z\rangle\langle z|,
$$

where $\phi(z)$ is in general a distribution. For $\hat{A} \in \mathcal{K}$, the nature of $\phi(z)$ can be more precisely specified: 


$$
\begin{aligned}
\tilde{\phi}(\tau, \sigma) & =\text { Fourier transform of } \phi(z) \\
& =\iint \mathrm{d} q \mathrm{~d} p \mathrm{e}^{i(\tau q-\sigma p)} \phi\left(\frac{q+i p}{\sqrt{2}}\right) \\
& =\exp \left\{\frac{1}{4}\left(\tau^{2}+\sigma^{2}\right)\right\} a(\tau, \sigma),
\end{aligned}
$$

where $a(\tau, \sigma)$ is the Weyl weight of $\hat{A}$ appearing in eq. (2.8).

The main aim of the rest of this material is to examine the possibility of such a diagonal representation in the case of generalized coherent states.

\section{Two approaches to generalized coherent states}

We now briefly outline the Klauder [1] and the Perelomov [2] forms of generalized coherent state systems.

\section{The Klauder form}

The framework used is the Hilbert space $\mathcal{H}$ of some quantum system, and a finite dimensional topological space $\mathcal{L}$ which plays the role of a 'label space'. There are however no primary operator structures used in the definition. For each point $\ell \in \mathcal{L}$ a generalized coherent state $|\ell\rangle \in \mathcal{H}$ is given in a one-to-one manner, obeying the following three conditions:

(i) $\quad \||\ell\rangle \|=1$

(ii) $|\ell\rangle$ is strongly continuous in $\ell$;

(iii) there is a volume element $\mathrm{d} \ell$ on $\mathcal{L}$

$$
\text { such that we have a resolution of identity }
$$

$$
\int_{\mathcal{L}} \mathrm{d} \ell|\ell\rangle\langle\ell|=1 \text { on } \mathcal{H},
$$

where the last equation holds in a weak sense of matrix elements. Such a triple $\{\mathcal{H}, \mathcal{L},|\ell\rangle\}$ is a system of generalized coherent states. In a sense this is a very spare and economical definition, but it is not constructive.

The Fock states $|n\rangle$ and the position eigenstates $|q\rangle$ both violate these conditions: the former is not continuous, the latter is not normalisable. Some of the interesting consequences are as follows [6]:

(a) For each $|\psi\rangle \in \mathcal{H}$ we have a bounded continuous 'wavefunction'

$$
\psi(\ell)=\langle\ell \mid \psi\rangle
$$

with the help of which we can write 


$$
\begin{aligned}
|\psi\rangle & =\int_{\mathcal{L}} \mathrm{d} \ell \psi(\ell)|\ell\rangle, \\
\langle\psi \mid \psi\rangle & =\int_{\mathcal{L}} \mathrm{d} \ell|\psi(\ell)|^{2} .
\end{aligned}
$$

For these wavefunctions there is no need to talk of Lebesgue square integrability, definitions up to sets of measure zero, and the like. Clearly every $\psi(\ell) \in L^{2}(\mathcal{L})$, and $\mathcal{H}$ is contained in $L^{2}(\mathcal{L})$ as a proper subset.

(b) There is a reproducing kernel

$$
K\left(\ell^{\prime} ; \ell\right)=\left\langle\ell^{\prime} \mid \ell\right\rangle
$$

which is continuous in both $\ell^{\prime}$ and $\ell$, and which obeys:

$$
\begin{aligned}
& K\left(\ell^{\prime} ; \ell\right)^{*}=K\left(\ell ; \ell^{\prime}\right) ; \\
& \int_{\mathcal{L}} \int_{\mathcal{L}} \mathrm{d} \ell^{\prime} \mathrm{d} \ell f\left(\ell^{\prime}\right)^{*} K\left(\ell^{\prime} ; \ell\right) f(\ell) \geq 0 ; \\
& \psi(\ell)=\int_{\mathcal{L}} \mathrm{d} \ell^{\prime} K\left(\ell ; \ell^{\prime}\right) \psi\left(\ell^{\prime}\right) ; \\
& |\ell\rangle=\int_{L} \mathrm{~d} \ell^{\prime} \mathcal{K}\left(\ell ; \ell^{\prime}\right)\left|\ell^{\prime}\right\rangle .
\end{aligned}
$$

Properties (i) and (ii) are the hermiticity and positive definiteness of $K\left(\ell^{\prime} ; \ell\right)$; property (iii) shows very clearly why $\mathcal{H}$ is a proper subset of $L^{2}(\mathcal{L})$; and property (iv) shows that the system of generalized coherent states is overcomplete.

In some cases, but not necessarily always, the overcompleteness leads to entire functions and characteristic sets playing a special role.

The possibility of an operator $\hat{A}$ being determined by its diagonal matrix elements $\langle\ell|\hat{A}| \ell\rangle$, and of an operator possessing a diagonal coherent state representation, get related in a useful way. Let us define two linear subspaces $\mathcal{K}_{1}$ and $\mathcal{K}_{2}$ in the second Hilbert space $\mathcal{K}$ of all Hilbert-Schmidt operators on $\mathcal{H}$ as follows:

$$
\begin{aligned}
& \left.\mathcal{K}_{1}=\left\{\hat{A} \in \mathcal{K}\left|\hat{A}=\int_{\mathcal{L}} \mathrm{d} \ell a(\ell)\right| \ell\right\rangle\langle\ell|, \text { some } a(\ell)\right\} \subset \mathcal{K} ; \\
& \mathcal{K}_{2}=\{\hat{A} \in \mathcal{K} \mid\langle\ell|\hat{A}| \ell\rangle=0, \text { all } \ell \in \mathcal{L}\} \subset \mathcal{K} .
\end{aligned}
$$

Then we find, with respect to the inner product (2.6) on $\mathcal{K}$ :

$$
\begin{aligned}
\mathcal{K}_{2} & =\mathcal{K}_{1}^{\perp}, \\
\mathcal{K} & =\mathcal{K}_{1} \oplus \mathcal{K}_{2} .
\end{aligned}
$$

Thus any operator $\hat{A} \in \mathcal{K}$ is uniquely expressible as the sum of a part $\hat{A}_{1} \in \mathcal{K}_{1}$ which possesses a diagonal representation, and a part $\hat{A}_{2} \in \mathcal{K}_{2}$ all of whose diagonal coherent 
state matrix elements vanish. Furthermore, for a nonzero $\hat{A}_{1} \in \mathcal{K}_{1},\left\langle\ell\left|\hat{A}_{1}\right| \ell\right\rangle$ cannot vanish identically; and for a nonzero $\hat{A}_{2} \in \mathcal{K}_{2}$, we have no diagonal coherent state representation. As further consequences, any $\hat{A} \in \mathcal{K}$ is determined by $\langle\ell|\hat{A}| \ell\rangle$ up to an element in $\mathcal{K}_{2}$; and any $\hat{A}_{1} \in \mathcal{K}_{1}$ is uniquely determined by $\left\langle\ell\left|\hat{A}_{1}\right| \ell\right\rangle$.

\section{The Perelomov form [7]}

In contrast to the previous setup, we now have a more specific operator framework assumed right at the start, in fact a quite elaborate one. We have a Hilbert space $\mathcal{H}$, a Lie group $G$, and a unitary irreducible representation (UIR) $\mathcal{U}(g), g \in G$, of $G$ on $\mathcal{H}$. We choose some normalised fiducial vector $\left|\psi_{0}\right\rangle \in \mathcal{H}$, with corresponding pure state density operator $\hat{\rho}_{0}=\left|\psi_{0}\right\rangle\left\langle\psi_{0}\right|$. Through actions by $G$ we generate the orbits of $\psi_{0}$ and $\hat{\rho}_{0}$ :

$$
\begin{aligned}
\vartheta\left(\psi_{0}\right) & =\left\{\psi(g)=\mathcal{U}(g) \psi_{0} \mid g \in G\right\} \subset \mathcal{H}, \\
\vartheta\left(\hat{\rho}_{0}\right) & =\left\{\hat{\rho}(g)=\mathcal{U}(g) \hat{\rho}_{0} \mathcal{U}(g)^{\dagger}=\psi(g) \psi(g)^{\dagger} \mid g \in G\right\} \subset \mathcal{K} .
\end{aligned}
$$

The orbit $\vartheta\left(\psi_{0}\right)$ of $\psi_{0}$ is embedded within the unit sphere in $\mathcal{H}$, and its real dimension is less than or equal to the dimension of $G$. The orbit $\vartheta\left(\hat{\rho}_{0}\right)$ of $\hat{\rho}_{0}$ is a subset of the set of pure state density operators on $\mathcal{H}$.

A generalized coherent state system is now defined as the collection of unit vectors $\psi(g)$ comprising the orbit $\vartheta\left(\psi_{0}\right)$; so it brings together $\mathcal{H}, G, \mathcal{U}(g)$ and $\psi_{0}$ in a special way.

The two orbits defined in (3.8) can be identified with coset spaces of $G$ with respect to two corresponding stability groups:

$$
\begin{aligned}
H_{0} & =\left\{g \in G \mid \mathcal{U}(g) \psi_{0}=\psi_{0}\right\} \subset G, \\
\vartheta\left(\psi_{0}\right) & \simeq G / H_{0} ; \\
H & =\left\{g \in G \mid \mathcal{U}(g) \psi_{0}=(\text { phase }) \psi_{0}\right\} \\
& =\left\{g \in G \mid \mathcal{U}(g) \hat{\rho}_{0} \mathcal{U}(g)^{\dagger}=\hat{\rho}_{0}\right\} \subset G, \\
\vartheta\left(\hat{\rho}_{0}\right) & \simeq G / H .
\end{aligned}
$$

The subgroup $H_{0}$ is the stability group of $\psi_{0}$ in the strict sense, while $H$ is the stability group of $\psi_{0}$ up to phases; their possible mutual relationships will be examined shortly.

We can make contact with the Klauder form in the following sense. As the correspondence between $\vartheta\left(\psi_{0}\right)$ and $G / H_{0}$ is one-to-one onto, we can identify the topological space $\mathcal{L}$ here as the coset space:

$$
\mathcal{L}=G / H_{0} .
$$

However the present generalised coherent state system would be also an instance of the earlier form only if a resolution of the identity can be established; this is not one of the requirements in the Perelomov form. We take up this aspect below.

The stability group $H_{0}$ is always an invariant subgroup of $H$. There are three possible interesting relationships between them, distinguished by the nature of the factor group $H / H_{0}$ : 


$$
\begin{aligned}
H / H_{0} & =\text { trivial, } H=H_{0}, \vartheta\left(\psi_{0}\right) \simeq \vartheta\left(\hat{\rho}_{0}\right) ; \\
H / H_{0} & =\text { discrete nontrivial, } \\
\vartheta\left(\psi_{0}\right) & =\text { discrete cover of } \vartheta\left(\hat{\rho}_{0}\right) ; \\
H / H_{0} & =U(1), \\
\vartheta\left(\psi_{0}\right) & \simeq \text { principal } U(1) \text { fibre bundle over } \vartheta\left(\hat{\rho}_{0}\right) .
\end{aligned}
$$

In case (i), the phase of $\psi_{0}$ cannot be altered by action by any element of $G$; in case (ii), only a discrete set of phase changes can be made; while in case (iii) the phase of $\psi_{0}$ can be altered by any amount upon action by $\mathcal{U}(g)$ for suitable $g \in G$. With quite simple finite dimensional examples one can easily realise all three situations.

Now we consider the possibility of a resolution of the identity. We assume we have a (left and right) translation invariant volume element $\mathrm{d} g$ over $G$. Then as the representation $\mathcal{U}(g)$ is irreducible, Schur's lemma implies that

$$
\begin{aligned}
\int_{G} \mathrm{~d} g|\psi(g)\rangle\langle\psi(g)| & =\int_{G} \mathrm{~d} g \hat{\rho}(g) \\
& =c \cdot 1,
\end{aligned}
$$

where $c$ is some constant. If $c$ is finite, we recover in full detail a system of generalized coherent states in the Klauder form. On the other hand if $c$ diverges, we have such a system in the Perelomov form, but not in the Klauder form. If $\mathcal{H}$ is finite dimensional, it is clear that $c$ will be finite; then a Perelomov system is a particular case of a Klauder system. In the case of infinite dimensional $\mathcal{H}$, the finiteness of $c$ will follow if the representation $\mathcal{U}(g)$ of $G$ happens to be 'square integrable'.

The possible resolution of the identity can be expressed in terms of integrations over the coset spaces as well. Let $q \in \vartheta\left(\psi_{0}\right)$ and $r \in \vartheta\left(\hat{\rho}_{0}\right)$ denote general points in these coset spaces. Then we can choose (local) coset representatives $\ell_{0}(q), \ell(r) \in G$ such that a general $g \in G$ can be written as a product in two ways:

$$
\begin{aligned}
g=\ell_{0}(q) h_{0} \\
=\ell(r) h, \\
h_{0} \in H_{0}, \quad h \in H .
\end{aligned}
$$

(Again locally, $\ell_{0}(q)$ is the product of $\ell(r)$ with a suitable trivial, discrete or continuous $U(1)$ element on the right.) From the invariant volume element $d g$ on $G$ we get reduced volume elements $\mathrm{d} q, \mathrm{~d} r$ on the coset spaces; and the Schur lemma reduces to

$$
\int_{G / H} \mathrm{~d} r \hat{\rho}(\ell(r))=c \cdot 1 .
$$

Even though in the Klauder sense we identify $\mathcal{L}$ with $G / H_{0}$, for the resolution of the identity it is more economical to integrate over the (smaller) coset space $G / H$.

In summary, only if $c<\infty$ does a general coherent state system in the Perelomov sense also constitute such a system in the Klauder sense. Thus one definition is not subsumed by the other in either direction. 


\section{Reinterpretation of standard coherent states and further examples}

We can easily recover the standard coherent states as instances of both the Klauder and the Perelomov systems. The relevant Hilbert space is $\mathcal{H}=\ell^{2}=L^{2}(\mathcal{R})$. To make contact with the Klauder form we identify the topological space $\mathcal{L}$ and the Klauder state $|\ell\rangle$ as follows:

$$
\begin{aligned}
\mathcal{L}=C, \ell & =z \in C, \\
|\ell\rangle & =|z\rangle=D(z)|0\rangle .
\end{aligned}
$$

The strong continuity condition and the resolution of the identity are both satisfied, and the reproducing kernel in this case is given in eq. (2.10).

To reach the Perelomov form we identify the Lie group $G$ as the Heisenberg-Weyl group (H-W group) with $\hat{q}$ and $\hat{p}$ as generators. In the relevant UIR, the elements of this group are realised as phase factors times the displacement operator $D(q, p)$ of eq. (2.4).

$$
\begin{aligned}
\mathcal{U}(q, p, \alpha) & =\mathrm{e}^{i \alpha} D(q, p), \alpha \in[0,2 \pi), q, p \in \mathcal{R} ; \\
\mathcal{U}\left(q^{\prime}, p^{\prime}, \alpha^{\prime}\right) \mathcal{U}(q, p, \alpha) & =\mathcal{U}\left(q^{\prime}+q, p^{\prime}+p, \alpha^{\prime}+\alpha+\frac{1}{2}\left(p^{\prime} q-q^{\prime} p\right) \bmod 2 \pi\right) .
\end{aligned}
$$

The fiducial vector $\psi_{0}$ for the Perelomov construction is $\psi_{0}=|0\rangle$, the ground or vacuum state in the Fock basis. The two stability groups are

$$
\begin{aligned}
H_{0} & =\{e\}, \\
H & =U(1)=\{\mathcal{U}(0,0, \alpha) \mid \alpha \in[0,2 \pi)\} .
\end{aligned}
$$

The orbit of $\psi_{0}$ is just the set of standard coherent states multiplied by phases:

$$
\begin{aligned}
& |z ; \alpha\rangle=\mathcal{U}(q, p, \alpha) \psi_{0}=\mathrm{e}^{i \alpha} D(z)|0\rangle, \\
& \vartheta\left(\psi_{0}\right)=\left\{\mathrm{e}^{i \alpha}|z>| z \in C, \alpha \in[0,2 \pi)\right\} .
\end{aligned}
$$

We are dealing here with the Stone-von Neumann UIR of the H-W group and this is a square integrable UIR (as we will soon see via the useful Moyal identity), so we have consistency with the known resolution of the identity, eq. (2.13).

Having seen that the standard coherent states are an instance of both the Klauder and the Perelomov systems, we now briefly describe some other examples of generalized coherent state systems.

(a) $G=H-W$ group, generic fiducial vector

In the Hilbert space $\mathcal{H}=L^{2}(\mathcal{R})$ we choose as fiducial vector $\psi_{0}$ a general normalised vector, not necessarily the ground state $|0\rangle$ of the Fock basis. Then the Perelomov system of GCS is

$$
\begin{aligned}
\left|z, \alpha ; \psi_{0}\right\rangle & =\mathcal{U}(q, p, \alpha)\left|\psi_{0}\right\rangle \\
& =\mathrm{e}^{i \alpha} D(z)\left|\psi_{0}\right\rangle=\mathrm{e}^{i \alpha}\left|z ; \psi_{0}\right\rangle,
\end{aligned}
$$

and this is a unit vector for all $z$, continuous in $z$ as well. Quite generally, for any $\psi_{0}$ one finds $H_{0}=\{e\}, H=U(1)$ exactly as in eq. (4.3). The resolution of the identity 
also holds; thanks to the general Moyal formula valid for any four (normalisable) vectors $\left|\varphi_{1}\right\rangle,\left|\varphi_{2}\right\rangle,\left|\varphi_{3}\right\rangle,\left|\varphi_{4}\right\rangle$ in $\mathcal{H}[8]$ :

$$
\int \frac{\mathrm{d}^{2} z}{\pi}\left\langle\varphi_{1}|D(z)| \varphi_{2}\right\rangle\left\langle\varphi_{4}\left|D(z)^{\dagger}\right| \varphi_{3}\right\rangle=\left\langle\varphi_{1} \mid \varphi_{3}\right\rangle\left\langle\varphi_{4} \mid \varphi_{2}\right\rangle .
$$

This is in fact a characterisation of the Stone-von Neumann UIR of the H-W group. Setting $\left|\varphi_{2}\right\rangle=\left|\varphi_{4}\right\rangle=\left|\psi_{0}\right\rangle$ here gives, for any $\left|\varphi_{1}\right\rangle$ and $\left|\varphi_{3}\right\rangle$,

$$
\begin{aligned}
\int \frac{\mathrm{d}^{2} z}{\pi}\left\langle\varphi_{1}|D(z)| \psi_{0}\right\rangle\left\langle\psi_{0}\left|D(z)^{\dagger}\right| \varphi_{3}\right\rangle & =\left\langle\varphi_{1} \mid \varphi_{3}\right\rangle, \\
\int \frac{\mathrm{d}^{2} z}{\pi}\left|z ; \psi_{0}\right\rangle\left\langle z ; \psi_{0}\right| & =1 \text { on } \mathcal{H} .
\end{aligned}
$$

This system is therefore both a Klauder and a Perelomov system of GCS.

The reproducing kernel of the Klauder formulation is

$$
\begin{aligned}
K\left(z^{\prime} ; z\right) & =\left\langle z^{\prime} ; \psi_{0} \mid z ; \psi_{0}\right\rangle \\
& =\left\langle\psi_{0}\left|D\left(z^{\prime}\right)^{\dagger} D(z)\right| \psi_{0}\right\rangle \\
& =\left\langle\psi_{0}\left|D\left(z-z^{\prime}\right)\right| \psi_{0}\right\rangle \mathrm{e}^{i} \operatorname{Im}{z^{\prime}}^{*} z
\end{aligned}
$$

so the expectation values of the displacement operators in the fiducial state play an important role. The following results are known to hold for any $\psi_{0}$ : the family of states $\left\{\left|z_{1} ; \psi_{0}\right\rangle \mid z \in C\right\}$ is always overcomplete. There exist characteristic sets in $C$ analogous to the case of ordinary or standard coherent states. And there is a very interesting result due to Klauder concerning the possibility of diagonal representations for general operators [3]:

$$
\begin{aligned}
\mathcal{K}_{2}=0 & \Leftrightarrow \text { every } \hat{A} \text { has a diagonal representation } \\
& \Leftrightarrow\left\langle\psi_{0}|D(z)| \psi_{0}\right\rangle \neq 0, \text { all } z \in C \\
& \Leftrightarrow K\left(z^{\prime} ; z\right) \neq 0, \text { all } z^{\prime}, z \in C .
\end{aligned}
$$

The choice $\left|\psi_{0}\right\rangle=|n\rangle, n=1,2, \ldots$, of the Fock basis has been studied long ago by Roy and Singh [9]. In this [10] case it is known that

$$
\langle n|D(z)| n\rangle=\mathrm{e}^{-\frac{1}{2}|z|^{2}} L_{n}\left(|z|^{2}\right),
$$

where $L_{n}(\cdot)$ is the Laguerre polynomial of order $n$. This polynomial has $n$ simple zeros on the positive real axis, so in the complex plane the quantity $\langle n|D(z)| n\rangle$ has $n$ concentric rings of zeros. We conclude that the condition (4.9) is not obeyed for $n \geq 1$, hence $\mathcal{K}_{2} \neq 0$ and for a general operator $\hat{A}$ we do not have a diagonal representation in these cases.

(b) $G=\mathrm{SU}(2)$

We consider the spin $j$ UIR $\mathcal{U}(\cdot)$ of SU(2), acting on a Hilbert space $\mathcal{H}$ of dimension $2 j+1$, where $j=0,1 / 2,1,3 / 2 \ldots$. (The UIR is faithful only when $j=1 / 2,3 / 2, \ldots$.) The following facts regarding the stability groups $H_{0}, H$ are generally known: 
(i) For $j=1 / 2$, namely in the defining UIR of $\mathrm{SU}(2)$, for any choice of $\psi_{0}$ we have:

$$
\begin{aligned}
H_{0} & =\{e\}, H=U(1) ; \\
\vartheta\left(\psi_{0}\right) & =\operatorname{SU}(2), \vartheta\left(\hat{\rho}_{0}\right)=S^{2} .
\end{aligned}
$$

(ii) For $j \geq 1$, if $\psi_{0}$ is a generic vector with no special properties, both $H_{0}$ and $H$ are trivial or discrete.

(iii) For $j \geq 1$, if $\psi_{0}=|m\rangle, m \neq 0$, an eigenvector of the third component $J_{3}$ of the $\mathrm{SU}(2)$ generators, (or any $\mathrm{SU}(2)$ transform of such an $|m\rangle$ ), then $H_{0}$ is trivial or discrete while $H=U(1)$.

(iv) For $j=$ integer $\geq 1$, if $\psi_{0}=|0\rangle$ (or any $\mathrm{SU}(2)$ transform of $|0\rangle$ ), then $H_{0}=H=$ $U(1)$.

Now make the specific choice $\psi_{0}=|j\rangle$, the 'highest weight state' in the spin $j$ UIR. The ensuing Perelomov family of GCS is the family of spin coherent states. Working directly and for simplicity on $G / H=S^{2}$, we identify the topological space $\mathcal{L}$ of Klauder with $S^{2}$. Then the spin coherent states are, using spherical polar variables to parametrise $S^{2}$ :

$$
\begin{aligned}
(\theta, \phi) \in S^{2}:|\theta, \phi\rangle & =\mathrm{e}^{-i \phi J_{3}} \mathrm{e}^{-i \theta J_{2}}|j\rangle \\
& =\mathrm{e}^{-i j \phi}(\cos \theta / 2)^{2 j} \mathrm{e}^{z J_{-}}\left|{ }_{j}\right\rangle, \\
z & =\mathrm{e}^{i \phi} \tan \theta / 2, \\
J_{-} & =J_{1}-i J_{2} .
\end{aligned}
$$

By expanding the exponential we find that $|\theta, \phi\rangle$ is a simple linear combination of $|m\rangle$ for $m=j, j-1, \ldots,-j$. Both the reproducing kernel and the resolution of the identity are easy to handle.

$$
\begin{aligned}
K\left(\theta^{\prime}, \phi^{\prime} ; \theta, \phi\right)= & \left\langle\theta^{\prime}, \phi^{\prime} \mid \theta, \phi\right\rangle \\
= & \mathrm{e}^{i j\left(\phi^{\prime}-\phi\right)}\left(\cos \theta^{\prime} / 2 \cos \theta / 2+\mathrm{e}^{-i\left(\phi^{\prime}-\phi\right)} \sin \theta^{\prime} / 2 \sin \theta / 2\right)^{2 j} ; \\
& \int_{S^{2}} \mathrm{~d} \cos \theta \mathrm{d} \phi|\theta, \phi\rangle\langle\theta, \phi|=\pi \cdot \frac{2^{2 j}}{2 j+1} \cdot 1 .
\end{aligned}
$$

So everything is in place and we have simultaneously a Klauder and a Perelomov system.

(c) $G=\overline{\mathrm{SU}(1,1)}$

Here we deal with the universal covering group $\overline{\mathrm{SU}(1,1)}$ of the group $\mathrm{SU}(1,1)$; the former covers the latter infinitely many times, and both are three dimensional. The situations that arise here are much more intricate than with the two previous cases. We begin with a brief recapitulation of the structure of the Lie algebra of SU(1,1), then proceed to the UIR's of interest.

We have three hermitian generators $J_{0}, K_{1}$ and $K_{2}$ obeying

$$
\left[J_{0}, K_{1}\right]=i K_{2},\left[J_{0}, K_{2}\right]=-i K_{1},\left[K_{1}, K_{2}\right]=-i J_{0} .
$$

The quadratic Casimir invariant is 


$$
Q=K_{1}^{2}+K_{2}^{2}-J_{0}^{2},
$$

and in a UIR we can work in a basis with $Q$ a number and $J_{0}$ diagonal.

The UIR's we look at are the so-called discrete positive and negative ones written as $D_{k}^{(+)}$and $D_{k}^{(-)}$respectively; here $k>0$ is a real parameter and $Q=k(1-k)$. In $D_{k}^{(+)}, J_{0}$ has the eigenvalues $k+n, n=0,1,2, \ldots$; while in $D_{k}^{(-)}$they are $-(k+n), n=0,1,2, \ldots$. In both cases there are no multiplicities. We can give a largely common treatment for $D_{k}^{(+)}$ and $D_{k}^{(-)}$. A common Hilbert space $\mathcal{H}_{k}$ suffices for both. A Fock-like basis for $\mathcal{H}_{k}$ consists of vectors $|k ; n\rangle, n=0,1,2, \ldots$, obeying

$$
\left\langle k ; n^{\prime} \mid k ; n\right\rangle=\delta_{n^{\prime} n} .
$$

Then in $D_{k}^{(+)}$we have the actions of the generators on these vectors given by

$$
\begin{aligned}
J_{0}|k ; n\rangle & =(k+n)|k ; n\rangle ; \\
\left(K_{1}+i K_{2}\right)|k ; n\rangle & =\sqrt{(n+1)(n+2 k)}|k ; n+1\rangle ; \\
\left(K_{1}-i K_{2}\right)|k ; n\rangle & =\sqrt{n(n-1+2 k)}|k ; n-1\rangle .
\end{aligned}
$$

Thus the lowering combination $K_{-}=K_{1}-i K_{2}$ annihilates $|k ; 0\rangle$. For $D_{k}^{(-)}$, in the same $\mathcal{H}_{k}$ and in the same basis $|k ; n\rangle$, we just replace

$$
\begin{aligned}
J_{0} & \rightarrow J_{0}^{\prime}=-J_{0} ; \\
K_{+}=K_{1}+i K_{2} & \rightarrow K_{+}^{\prime}=K_{1}^{\prime}+i K_{2}^{\prime}=K_{-}=K_{1}-i K_{2} ; \\
K_{-}=K_{1}-i K_{2} & \rightarrow K_{-}^{\prime}=K_{1}^{\prime}-i K_{2}^{\prime}=K_{+}=K_{1}+i K_{2} .
\end{aligned}
$$

Then $J_{0}^{\prime}, K_{1}^{\prime}, K_{2}^{\prime}$ generate the UIR $D_{k}^{(-)}$. Clearly the spectrum of $J_{0}^{\prime}$ is $-(k+n), n=$ $0,1, \ldots$, and now the raising combination $K_{+}^{\prime}$ annihilates $|k ; 0\rangle$. Note that the value of $Q$ does not distinguish $D_{k}^{(+)}$from $D_{k}^{(-)}$.

In this setting, two distinct types of GCS have been explored. One is to generalise the notion of annihilation operator eigenstates occurring in the standard coherent states, the other is in the spirit of the Perelomov method. The former is due to Barut and Girardello [11].

\section{Barut-Girardello construction in $D_{k}^{( \pm)}$}

These GCS are defined in the case of $D_{k}^{(+)}$to be the normalized right eigenstates of the lowering operator $K_{-}$. The states themselves, the reproducing kernel and the resolution of the identity are all explicitly given:

$$
\begin{aligned}
K_{-}|z\rangle & =z|z\rangle, z \in C, \\
|z\rangle & =\left\{{ }_{0} F_{1}\left(2 k ;|z|^{2}\right)\right\}^{-1 / 2} \sum_{n=0}^{\infty} \sqrt{\Gamma(2 k) / n ! \Gamma(n+2 k)} z^{n}|k ; n\rangle ; \\
\left\langle z^{\prime} \mid z\right\rangle & ={ }_{0} F_{1}\left(2 k ; z^{\prime *} z\right) / \sqrt{{ }_{0} F_{1}\left(2 k ;\left|z^{\prime}\right|^{2}\right)_{0} F_{1}\left(2 k ;|z|^{2}\right)} ;
\end{aligned}
$$




$$
\begin{gathered}
\int_{C} \frac{\mathrm{d}^{2} z}{\pi} \sigma\left(|z|^{2}\right)|z\rangle\langle z|=1 \text { on } \mathcal{H}_{k}, \\
\sigma(r)=\frac{2}{\Gamma(2 k)}{ }_{0} F_{1}(2 k ; r) r^{k-1 / 2} K_{\frac{1}{2}-k}(2 \sqrt{r}), k>0 .
\end{gathered}
$$

For this GCS system, no group action or fiducial vector choice is involved, so we are not dealing with a Perelomov system. We have a system in the Klauder sense with $\mathcal{L}$ identified with the complex plane $C$.

In the UIR $D_{k}^{(-)}$, as $\mathcal{H}_{k}$ is the same and $K_{-}=K_{+}^{\prime}$, the same states as given in eq. (4.19) are right eigenstates of the raising operator $K_{+}^{\prime}$, there are no changes at all.

\section{Perelomov type GCS in $D_{k}^{( \pm)}$}

Now use of the $\overline{\mathrm{SU}(1,1)}$ action on $\mathcal{H}_{k}$ becomes relevant, and some fiducial vector choice has to be made. In the case of $D_{k}^{(+)}$for any $k>0$, we choose $\psi_{0}$ to be $|k ; 0\rangle$, the eigenstate of $J_{0}$ with the minimum eigenvalue $k$. It is clear that in general $H_{0}=\{e\}$ and $H_{1}=\overline{U(1)} \simeq \mathcal{R}$, except that if $k$ is rational, $H_{1}$ reduces to $U(1)$. The GCS are generated by action of $\overline{\mathrm{SU}(1,1)}$ elements on $|k ; 0\rangle$. It is convenient to label them with a complex number $w$ within the unit disc $\mathcal{D}$ in $C$. Their definitions and the reproducing kernel turn out to be:

$$
\begin{aligned}
\left|\Psi^{(+)}(w)\right\rangle & =\mathrm{e}^{-i\left(\alpha_{1} K_{1}+\alpha_{2} K_{2}\right)}|k ; 0\rangle \\
& =\left(1-|w|^{2}\right)^{k} \sum_{n=0}^{\infty} \sqrt{\frac{\Gamma(n+2 k)}{n ! \Gamma(2 k)}} w^{n}|k ; n\rangle, \\
w & =\frac{\left(\alpha_{1}+i \alpha_{2}\right)}{\left|\alpha_{\perp}\right|} \tanh \left(\frac{1}{2}\left|\alpha_{\perp}\right|\right) \in \mathcal{D} ; \\
K\left(w^{\prime} ; w\right) & =\left\langle\Psi^{(+)}\left(w^{\prime}\right) \mid \Psi^{(+)}(w)\right\rangle \\
& =\left(1-\left|w^{\prime}\right|^{2}\right)^{k}\left(1-|w|^{2}\right)^{k}\left(1-w^{\prime *} w\right)^{-2 k} .
\end{aligned}
$$

So for any $k>0$ we have here a well-defined Perelomov system. When we seek for a resolution of the identity, however, we see that is possible only for $k>1 / 2$ and not for $0<k \leq \frac{1}{2}$ :

$$
\begin{gathered}
\int_{\mathcal{D}} \mathrm{d}^{2} w \sigma\left(|w|^{2}\right) \quad\left|\Psi^{(+)}(w)\right\rangle\left\langle\Psi^{(+)}(w)\right|=1 \text { on } \mathcal{H}_{k}, \\
\sigma(r)=\frac{1}{\pi} \cdot \frac{2 k-1}{(1-r)^{2}}, k>\frac{1}{2} .
\end{gathered}
$$

Thus these Perelomov type GCS are simultaneously Klauder type GCS only if $k>1 / 2$, with the identification $\mathcal{L}=\mathcal{D} \subset C$.

In the case of $D_{k}^{(-)}$acting on the same $\mathcal{H}_{k}$, we have very similar results going with the choice $\psi_{0}=|k ; 0\rangle$, and with the replacement of $w$ by $w^{*}$. 
(d) Metaplectic oscillator representation of $\overline{\mathrm{SU}(1,1)}$

The results here are subsumed under what we have described above but merit brief separate mention. The unitary representation $(U R)$ of $\overline{\mathrm{SU}(1,1)}$ we are concerned with here is a reducible one, being the direct sum of $D_{1 / 4}^{(-)}$and $D_{3 / 4}^{(-)}$. In terms of the annihilation and creation operators $\hat{a}, \hat{a}^{+}$for a single degree of freedom, the generators are

$$
J_{0}=-\frac{1}{2} \hat{a}^{\dagger} \hat{a}-\frac{1}{4}, K_{1}=\frac{i}{4}\left(\hat{a}^{\dagger^{2}}-\hat{a}^{2}\right), K_{2}=-\frac{1}{4}\left(\hat{a}^{\dagger^{2}}+\hat{a}^{2}\right) .
$$

The Casimir operator has the numerical value $Q=+3 / 16$, so both $k=\frac{1}{4}$ and $k=\frac{3}{4}$ are involved. In terms of the Fock basis $|n\rangle$ for the Hilbert space $\mathcal{H}$, the basis vectors $|1 / 4 ; n\rangle$ and $|3 / 4 ; n\rangle$ supporting the UIR's $D_{1 / 4}^{(-)}, D_{3 / 4}^{(-)}$respectively are identified thus:

$$
\begin{aligned}
\mathcal{H} & =\operatorname{Sp}\{|n\rangle \mid n=0,1,2, \ldots,\} \\
& =\mathcal{H}^{(+)} \oplus \mathcal{H}^{(-)} ; \\
\mathcal{H}^{(+)} & =\operatorname{Sp}\{|1 / 4 ; n\rangle \mid n=0,1,2, \ldots,\}, \\
|1 / 4 ; n\rangle & =|2 n\rangle ; \\
\mathcal{H}^{(-)} & =\operatorname{Sp}\{|3 / 4 ; n\rangle \mid n=0,1,2, \ldots,\}, \\
|3 / 4 ; n\rangle & =|2 n+1\rangle .
\end{aligned}
$$

Within each of $\mathcal{H}^{(+)}$and $\mathcal{H}^{(-)}$, we have both the Barut-Girardello type of GCS, namely

\begin{tabular}{|c|c|c|c|}
\hline & $D_{k}^{(+)}, k>0$ & $D_{k}^{(-)}, k>0$ & Remarks \\
\hline $\begin{array}{l}\text { Barut-Girardello } \\
\text { states }\end{array}$ & $K_{-}|z\rangle=z|z\rangle$ & $K_{+}^{\prime}|z\rangle=z|z\rangle$ & $\begin{array}{l}\text { Klauder system } \\
\text { for all } k>0 ; \text { not a } \\
\text { Perelomov system }\end{array}$ \\
\hline Perelomov states & $\begin{array}{c}\psi_{0}=|k ; 0\rangle, J_{0}=k: \\
\operatorname{GCS}=\left|\Psi^{(+)}(w)\right\rangle \\
w \in \mathcal{D}\end{array}$ & $\begin{array}{c}\psi_{0}=|k ; 0\rangle, J_{0}^{\prime}=-k \\
\operatorname{GCS}=\left|\Psi^{(-)}(w)\right\rangle \\
w \in \mathcal{D}\end{array}$ & $\begin{array}{l}\text { Resolution of identity, } \\
\text { i.e., Klauder system, } \\
\text { only if } k>1 / 2\end{array}$ \\
\hline $\begin{array}{l}\text { Metaplectic } \\
\text { oscillator } \\
\text { representation }\end{array}$ & $\begin{array}{c}k=1 / 4: \psi_{0}=|0\rangle_{\text {Fock }} \\
k=3 / 4: \psi_{0}=|1\rangle_{\text {Fock }}\end{array}$ & & $\begin{array}{l}\text { Perelomov system, } \\
\text { not Klauder system } \\
\text { Perelomov and } \\
\text { Klauder system }\end{array}$ \\
\hline
\end{tabular}
eigenstates of $K_{+}$; and the Perelomov family of GCS based on the choice of fiducial vector $\psi_{0}$ as $|1 / 4 ; 0\rangle$ and $|3 / 4 ; 0\rangle$ respectively. However, as seen earlier in eq. (4.21), the resolution of the identity is not available in the Perelomov system in $\mathcal{H}^{(+)}$. The treatments of GCS in the continuous series UIR's of $\overline{\mathrm{SU}(1,1)}$, and in the UR's of $S L(2, C)$, may be found in the monograph of Perelomov [7].

The $\overline{\mathrm{SU}(1,1)}$ results assembled above can be displayed in a table: 


\section{A new approach to the diagonal representation problem [12]}

We now outline a new approach to finding necessary and sufficient conditions for the existence of a diagonal representation for all operators $\hat{A} \in \mathcal{K}$, i.e., for having $\mathcal{K}_{2}=0$. This will be within the Perelomov framework for GCS, and uses both Clebsch-Gordan theory and the theory of induced representations of groups.

For definiteness we assume that $G$ is a compact Lie group, so that all its UIR's are finite dimensional. We will use the symbol $J$, which is in general actually a collection of several independent (discrete) labels, to denote the various UIR's of $G$. Thus we shall say that the UIR $\mathcal{D}^{(J)}$ of $G$ operates on the (finite dimensional) Hilbert space $\mathcal{H}^{(J)}$, and in a suitable basis the matrices of the UIR have elements $\mathcal{D}_{M^{\prime} M}^{(J)}(g)$, with the 'magnetic' quantum numbers $M^{\prime}$ and $M$ again each standing for several independent labels. Similarly, for the subgroup $H \subset G$ which will arise later, we have the UIR $D^{(j)}$ with matrix elements $D_{m^{\prime} m}^{(j)}(h)$ operating on the Hilbert space $\Im^{(j)}$. We will mainly work on the coset space $G / H$, denoting a general point of it by the symbol $r \in G / H$. For a choice of a (local) coset representative we use the notation $\ell(r)$ as in eq. (3.13), so any $g \in G$ is expressible as the product

$$
g=\ell(r) h, \quad g \in G, \quad r \in G / H, \quad h \in H .
$$

To start the Perelomov GCS construction we begin with the UIR $\mathcal{D}^{\left(J_{0}\right)}$ of $G$ on $\mathcal{H}^{\left(J_{0}\right)}$, and pick a fiducial vector $\psi_{0} \in \mathcal{H}^{\left(J_{0}\right)}$ having $H$ as its stability group up to phases. Then we have the GCS and their projections given by

$$
\begin{aligned}
\psi(g) & =\mathcal{D}^{\left(J_{0}\right)}(g) \psi_{0}, \\
\hat{\rho}(g) & =\psi(g) \psi(g)^{\dagger} \\
& =\mathcal{D}^{\left(J_{0}\right)}(g) \hat{\rho}_{0} \mathcal{D}^{\left(J_{0}\right)}(g)^{-1} .
\end{aligned}
$$

These projections have the twin properties

$$
\begin{aligned}
& h \in H: \mathcal{D}^{\left(J_{0}\right)}(h) \hat{\rho}_{0} \mathcal{D}^{\left(J_{0}\right)}(h)^{-1}=\hat{\rho}_{0} ; \\
& \mathcal{D}^{\left(J_{0}\right)}\left(g^{\prime}\right) \hat{\rho}(g) \mathcal{D}^{\left(J_{0}\right)}\left(g^{\prime}\right)^{-1}=\hat{\rho}\left(g^{\prime} g\right) ;
\end{aligned}
$$

which together imply, using the representation (5.1), that $\hat{\rho}(g)$ is actually an operator valued function on the coset space $G / H$ :

$$
\begin{aligned}
\hat{\rho}(g) & =\mathcal{D}^{\left(J_{0}\right)}(g) \hat{\rho}_{0} \mathcal{D}^{\left(J_{0}\right)}(g)^{-1} \\
& =\mathcal{D}^{\left(J_{0}\right)}(\ell(r) h) \hat{\rho}_{0} \mathcal{D}^{\left(J_{0}\right)}(\ell(r) h)^{-1} \\
& =\mathcal{D}^{\left(J_{0}\right)}(\ell(r)) \hat{\rho}_{0} \mathcal{D}^{\left(J_{0}\right)}(\ell(r))^{-1} \\
& =\hat{\rho}(\ell(r)), \\
\text { i.e., } \hat{\rho}(g) & =\hat{\rho}(\ell(r)) \equiv \hat{\rho}(r) .
\end{aligned}
$$

Thus the 'independent parts' of $\hat{\rho}(g)$ are just the $\hat{\rho}(r)$ defined in the last line above. On these $\hat{\rho}(r)$ the action of $G$ by conjugation is expressed in terms of the action of $G$ on $G / H$ via point transformations:

Pramana - J. Phys., Vol. 56, Nos 2 \& 3, Feb. \& Mar. 2001 


$$
\mathcal{D}^{\left(J_{0}\right)}(g) \hat{\rho}(r) \mathcal{D}^{\left(J_{0}\right)}(g)^{-1}=\hat{\rho}(g r) .
$$

We now pose the two questions:

(i) Which operators $\hat{A}$ on $\mathcal{H}^{\left(J_{0}\right)}$ arise by considering integrals of the form

$$
\hat{A}=\int_{G / H} \mathrm{~d} r a(r) \hat{\rho}(r)
$$

for all possible (locally integrable) choices of complex weight functions $a(r)$ ?

(ii) What are the necessary and sufficient conditions for $\mathcal{K}_{2}=0$, ie., for all operators $\hat{A}$ to have the diagonal representation (5.6)? We will answer these questions below; first we set up some preliminaries.

Even before the fiducial vector $\psi_{0}$ is chosen and the GCS $\psi(g)$ along with their projections $\hat{\rho}(g)$ are constructed, we know from Clebsch-Gordan (CG) theory how to construct an orthonormal basis of unit tensors for $\mathcal{K}^{\left(J_{0}\right)}$, the Hilbert space of operators on $\mathcal{H}^{\left(J_{0}\right)}$. Let the reduction of the direct product $\mathcal{D}^{\left(J_{0}\right)} \times \mathcal{D}^{\left(J_{0}\right)^{*}}$ read as follows:

$$
\begin{aligned}
\mathcal{D}^{\left(J_{0}\right)} \times \mathcal{D}^{\left(J_{0}\right)^{*}} & =\sum_{j} \oplus n_{J} \mathcal{D}^{(J)}, \\
n_{J} & =0,1,2, \ldots,
\end{aligned}
$$

where $n_{J}$ is the multiplicity of occurrence of the $J$ th UIR in the reduction. For each such $J$, we have a set of unit tensor operators $\mathcal{U}_{M}^{J \Lambda}$ carrying a multiplicity label $\Lambda$ taking values $\Lambda=1,2, \ldots, n_{J}$. Each $\mathcal{U}_{M}^{J \Lambda}$ is an operator on $\mathcal{H}^{\left(J_{0}\right)}$, therefore an element of $\mathcal{K}^{\left(J_{0}\right)}$; and the collection obeys

$$
\begin{aligned}
\mathcal{D}^{\left(J_{0}\right)}(g) \mathcal{U}_{M}^{J \Lambda} \mathcal{D}^{\left(J_{0}\right)}(g)^{-1} & =\sum_{M^{\prime}} \mathcal{D}_{M^{\prime} M}^{(J)}(g) \mathcal{U}_{M^{\prime}}^{J \Lambda} \\
\operatorname{Tr}\left(\mathcal{U}_{M^{\prime}}^{J^{\prime} \prime^{\dagger}} \mathcal{U}_{M}^{J \Lambda}\right) & \equiv\left(\mathcal{U}_{M^{\prime}}^{J^{\prime} \Lambda^{\prime}}, \mathcal{U}_{M}^{J \Lambda}\right) \\
& =\delta_{\Lambda^{\prime} \wedge} \delta_{J^{\prime} J} \delta_{M^{\prime} M}
\end{aligned}
$$

Naturally for a particular $J$ if $n_{J}>1$, we have considerable freedom in the choice of $\mathcal{U}_{M}^{J \Lambda}$ as unitary linear combinations over $\Lambda$ can always be made. Finally, any $\hat{A} \in \mathcal{K}^{\left(J_{0}\right)}$ has a unique expansion

$$
\begin{aligned}
\hat{A} & =\sum_{\Lambda J M} a_{\Lambda J M} \mathcal{U}_{M}^{J \Lambda}, \\
\|\hat{A}\|^{2} & =\operatorname{Tr}\left(\hat{A}^{\dagger} \hat{A}\right)=\sum_{\Lambda J M}\left|a_{\Lambda J M}\right|^{2} .
\end{aligned}
$$

All this information is therefore in our hands as soon as the UIR $\mathcal{D}^{\left(J_{0}\right)}$ is chosen, prior to choice of $\psi_{0}$ etc. Now we wish to know whether any $\hat{A}$ can also be expanded as an integral over $\hat{\rho}(r)$ as in eq. (5.6). For this we must clearly determine: given the projections $\hat{\rho}(r)$ transforming under $G$ according to eq. (5.5), which of the unit tensors $\mathcal{U}_{M}^{J \Lambda}$ with what multiplicities can be extracted from $\hat{\rho}(r)$ ? This question can be answered by carrying 
out harmonic analysis of $\hat{\rho}(r)$, based on the theory of induced representations [13]. We therefore briefly sketch the latter at this point.

Starting from any UIR $D^{(j)}$ of $H$ on the space $\Im^{(j)}$, by the inducing construction we obtain a specific UR $\mathcal{U}(g)$ of $G$ on a specially constructed Hilbert space $\mathcal{H}$ (for simplicity we avoid attaching any labels to this $\mathcal{H}$ ). Elements - 'vectors' - in $\mathcal{H}$ are functions $\psi$ on $G$ with values in $\Im^{(j)}$ and obeying a covariance condition in which the UIR $D^{(j)}$ of $H$ plays a role

$$
\begin{aligned}
& g \in G: \psi(g) \in \Im^{(j)} \\
& h \in H: \psi(g h)=D^{(j)}(h)^{\dagger} \psi(g) .
\end{aligned}
$$

Thus for such $\psi$ only the 'values' of $\psi(\ell(r))$ need be independently given, for then $\psi(g)$ for any $g$ is known. The inner product in $\mathcal{H}$ is then defined to be

$$
\begin{aligned}
\|\psi\|_{\mathcal{H}}^{2} & =\int_{G} \mathrm{~d} g(\psi(g), \psi(g))_{\Im(j)} \\
& =\int_{G / H} \mathrm{~d} r(\psi(\ell(r)), \psi(\ell(r)))_{\Im^{(j)}} .
\end{aligned}
$$

The UR $\mathcal{U}\left(g^{\prime}\right)$ of $G$ induced from the UIR $D^{(j)}$ of $H$ now acts in this manner on any $\psi \in \mathcal{H}$ :

$$
\left(\mathcal{U}\left(g^{\prime}\right) \psi\right)(g)=\psi\left(g^{\prime^{-1}} g\right) .
$$

The point to notice is that the covariance condition (5.10) on $\psi$ and $G$ action on $\psi$ do not come in each other's way as one acts on the right and the other on the left of the argument $g$ of $\psi$. In other words, the operators $\mathcal{U}(g)$ are well defined on $\mathcal{H}$, since they preserve the covariance condition; and they are of course also unitary with respect to the inner product (5.11) on $\mathcal{H}$. This induced UR of $G$ is in general reducible and the question arises as to its UIR contents and multiplicities: which UIR's $\mathcal{D}^{(J)}$ of $G$ occur and how often? The answer to this is contained in the beautiful Frobenius-Mackey reciprocity theorem [13].

The UR $\mathcal{U}(g)$ of $G$, induced from the UIR $D^{(j)}$ of the subgroup $H \subset G$, contains the UIR $\mathcal{D}^{(J)}$ of $G$ as often as $\mathcal{D}^{(J)}$ contains the UIR $D^{(j)}$ of $H$ upon restriction to $H$.

To apply this to the problem of finding out the irreducible tensor operator contents of $\hat{\rho}(r)$, in view of the first of eq. (5.3), we need to look at the induced UR $\mathcal{U}(g)$ of $G$ coming from the trivial UIR $(j=0)$ of $H$. This UR of $G$ is defined on the space $L^{2}(G / H)$ of square integrable complex valued $c$-number functions (not vectors!) of $r \in G / H$ :

$$
L^{2}(G / H)=\left\{\left.f(r) \in C\left|\|f\|^{2}=\int_{G / H} \mathrm{~d} r\right| f(r)\right|^{2}\right\} .
$$

To match the law of $G$ action on $\hat{\rho}(r)$ given in eq. (5.5), we make $G$ act on such functions $f(r)$ by

$$
(\mathcal{U}(g) f)(r)=f\left(g^{-1} r\right)
$$


Now this UR of $G$ on $L^{2}(G / H)$ contains the UIR $\mathcal{D}^{(J)}$ of $G$ as often as $\mathcal{D}^{(J)}$ contains scalars with respect to $H$. Let this multiplicity label be written as $\lambda$, to be carefully distinguished from $\Lambda$ appearing in eq. (5.8): they have quite different origins!

A complete set of 'spherical harmonics' on $G / H$ can therefore be written as $Y_{M}^{J \lambda}(r)$ : $\lambda$ labels the many occurrences of the same UIR $J$ of $G$ among functions on $G / H$. They obey the transformation law

$$
Y_{M}^{J \lambda}\left(g^{-1} r\right)=\sum_{M^{\prime}} \mathcal{D}_{M M^{\prime}}^{(J)}(g) Y_{M^{\prime}}^{J \lambda}(r)
$$

They form an orthonormal set of functions in $L^{2}(G / H)$ :

$$
\int_{G / H} \mathrm{~d} r Y_{M^{\prime}}^{J^{\prime} \lambda^{\prime}}(r)^{*} Y_{M}^{J \lambda}(r)=\delta_{J^{\prime} J} \delta_{\lambda^{\prime} \lambda} \delta_{M^{\prime} M} .
$$

And their completeness means that for any $f(r) \in L^{2}(G / H)$ we have

$$
\begin{aligned}
f(r) & =\sum_{J \lambda M} f_{J \lambda M} Y_{M}^{J \lambda}(r), \\
\|f\|^{2} & =\sum_{J \lambda M}\left|f_{J \lambda M}\right|^{2} .
\end{aligned}
$$

We are now in a position to perform the harmonic analysis of the operators $\hat{\rho}(r)$ and project out their irreducible tensor contents:

$$
\begin{aligned}
& \hat{\rho}(r)=\sum_{J \lambda M} \hat{\rho}_{M}^{J \lambda} Y_{M}^{J \lambda}(r), \\
& \hat{\rho}_{M}^{J \lambda}=\int_{G / H} \mathrm{~d} r Y_{M}^{J \lambda}(r)^{*} \hat{\rho}(r) .
\end{aligned}
$$

And by combining eqs (5.5), (5.15) and (5.18) we see that these $\hat{\rho}_{M}^{J \lambda}$ are indeed tensor operators of the indicated type:

$$
\mathcal{D}^{\left(J_{0}\right)}(g) \hat{\rho}_{M}^{J \lambda} \mathcal{D}^{\left(J_{0}\right)}(g)^{-1}=\sum_{M^{\prime}} \mathcal{D}_{M^{\prime} M}^{(J)}(g) \hat{\rho}_{M^{\prime}}^{J \lambda}
$$

We infer that any operator $\hat{A}$ expressible as an integral over $\hat{\rho}(r)$, namely any $\hat{A} \in \mathcal{K}_{1}$, is some linear combination of $\hat{\rho}_{M}^{J \lambda}$. The question is whether this is true for all $\hat{A} \in \mathcal{K}$, that is, whether $\mathcal{K}_{2}=0$.

It is in any case clear that as on the one hand, the unit tensors $\mathcal{U}_{M}^{J \Lambda}$ do form a complete set of operators on $\mathcal{H}^{\left(J_{0}\right)}$, and on the other hand the 'components' $\hat{\rho}_{M}^{J \lambda}$ projected out from $\hat{\rho}(r)$ are tensor operators of the indicated type, there must necessarily be linear relations having the following general structure [12]:

$$
\hat{\rho}_{M}^{J \lambda}=\sum_{\Lambda} \pi(J)^{\lambda} \wedge \mathcal{U}_{M}^{J \Lambda}
$$


Here for each $J, \pi(J)$ is a rectangular matrix made up of certain specific Clebsch-Gordan coefficients. The row label $\lambda$ has as many values as the multiplicity of occurrence of the UIR $\mathcal{D}^{(J)}$ of $G$ in the induced UR of $G$ given earlier, and this is the same as the multiplicity of occurrence of $H$-scalar states within this UIR $\mathcal{D}^{(J)}$. On the other hand the column label $\Lambda$ has as many values as the multiplicity of occurrence of the UIR $\mathcal{D}^{(J)}$ in the Clebsch-Gordan decomposition of the direct product $\mathcal{D}^{\left(J_{0}\right)} \times \mathcal{D}^{\left(J_{0}\right)^{*}}$. To orient oneself the following remark may be helpful: for compact $G$ and finite dimensional $\mathcal{D}^{(J)}$, the number of operators $\mathcal{U}_{M}^{J \Lambda}$ is necessarily finite, while (in any nontrivial situation) the number of independent spherical functions $Y_{M}^{J \lambda}(r)$ is always infinite. As a result all but a finite number of the operators $\hat{\rho}_{M}^{J \lambda}$ must actually vanish.

We can now see that the necessary and sufficient conditions for the existence of the diagonal representation for all operators $\hat{A} \in \mathcal{K}$ are as follows:

For each UIR $J$ of $G$, the number of values of the multiplicity label $\lambda$ must be greater than or equal to the number of values of the label $\Lambda$.

The rectangular matrix $\pi(J)$ must have maximal rank ie., its rank must be equal to the number of values of $\Lambda$.

If these two conditions are fulfilled, then (and only then) the unit tensors $\mathcal{U}_{M}^{J \Lambda}$ can be recovered from the set $\hat{\rho}_{M}^{J \lambda}$, i.e. eq. (5.20) can be inverted, and we obtain a diagonal representation for every operator $\hat{A}$. But especially in the finite dimensional case, as is obvious, this representation is highly nonunique.

We mention here a few examples to illustrate this result, omitting details:

\section{Examples}

(i) $G=\mathrm{SU}(2), \mathcal{D}^{\left(J_{0}\right)}=\operatorname{spin} J_{0}$ UIR for $J_{0} \geq 1$ :

If $\psi_{0}$ is a generic vector in the representation space $\mathcal{H}^{\left(J_{0}\right)}$, both $H_{0}$ and $H$ are trivial; a detailed examination shows that conditions (5.21) are obeyed and the diagonal representation exists.

(ii) $G=\mathrm{SU}(2), \mathcal{D}^{\left(J_{0}\right)}=\operatorname{spin} J_{0}$ UIR for integral $J_{0} \geq 1$ :

If $\psi_{0}$ is an eigenvector of the generator $J_{3}$ of $S O(2)$ rotations with eigenvalue zero, i.e. $\psi_{0}=\left|J_{0}, 0\right\rangle$ (or any $\mathrm{SU}(2)$ transform thereof) then conditions (5.21) are not obeyed, so we do not have the diagonal representation for general operators $\hat{A}$. Here both $H_{0}$ and $H$ are $U(1)$.

(iii) $G=\mathrm{SU}(2), \mathcal{D}^{\left(J_{0}\right)}=\operatorname{spin} J_{0}$ UIR for $J_{0} \geq 1 / 2$ :

If $\left.\overline{\psi_{0}=\mid J_{0},} M_{0}\right\rangle$ for some $M_{0} \neq 0$, conditions (5.21) are obeyed, and the diagonal representation exists. Here $H_{0}$ is trivial while $H$ is $U(1)$.

(iv) $G=\mathrm{SU}(3), \mathcal{D}^{\left(J_{0}\right)}=8$-dimensional octet or adjoint UIR:

If we choose $\psi_{0}$ to be the $\Sigma^{0}$ state in the language of particle physics, invariant under the diagonal $U(1) \times U(1)$ subgroup of $\mathrm{SU}(3)$, then $H_{0}=H=U(1) \times U(1)$. Conditions (5.21) fail and we do not get the diagonal representation. 
(v) $G=\mathrm{SU}(3), \mathcal{D}^{\left(J_{0}\right)}=8$-dimensional octet or adjoint UIR:

If we choose $\psi_{0}$ to be the $\Lambda$ state in the language of particle physics, invariant under the $U(2)$ subgroup of SU(3), then $H_{0}=H=U(2)$; once again conditions (5.21) fail and we do not get the diagonal representation.

(vi) $G=H-W$ group:

For any choice of $\psi_{0} \in \mathcal{H}$, direct analysis along the lines of the present work shows that

$$
\mathcal{K}_{2}=0 \Leftrightarrow\left\langle\psi_{0}|D(z)| \psi_{0}\right\rangle \neq 0 \text { for all } z \in C .
$$

This is just the Klauder criterion (4.9) recovered now via Clebsch-Gordan theory and the reciprocity theorem for induced representations. Assuming (5.22) is obeyed, we find that we can pass from the Weyl representation of an operator $\hat{A}$ to its diagonal representation using GCS based upon the fiducial vector $\psi_{0}$ as follows:

$$
\begin{aligned}
\hat{A} & =\iint \mathrm{d} q \mathrm{~d} p a(q, p) D(q, p) \\
& =\iint \frac{\mathrm{d}^{2} z}{\pi} \phi(z)\left|z ; \psi_{0}\right\rangle\left\langle z ; \psi_{0}\right|, \\
\left|z ; \psi_{0}\right\rangle & =D(z)\left|\psi_{0}\right\rangle ; \\
\text { Fourier transform of } \phi(z) & =a(q, p) /\left\langle\psi_{0}|D(q, p)| \psi_{0}\right\rangle^{*} .
\end{aligned}
$$

In the case of the standard coherent states when $\psi_{0}=|0\rangle$ of the Fock basis, we know that

$$
\langle 0|D(q, p)| 0\rangle=\mathrm{e}^{-\frac{1}{4}\left(q^{2}+p^{2}\right)}
$$

and we recover eq. (2.18). For general $\psi_{0}$, as we are dealing with a square integrable representation, $\left\langle\psi_{0}|D(q, p)| \psi_{0}\right\rangle$ must vanish sufficiently rapidly as $q, p \rightarrow \infty$ to be square integrable; this shows from eq. (5.23) that the weight function $\phi(z)$ always has the character of a distribution of some class determined by the choice of $\psi_{0}$.

\section{Concluding comments}

The methods we have outlined based on Clebsch-Gordan and induced representation theory give us a good grasp of operator aspects of GCS generated via the Perelomov method. It is gratifying that we have been able to state explicitly necessary and sufficient conditions for the existence of a diagonal generalised coherent state representation for general operators. Even in the well-studied case of the H-W group we are able to appreciate known results in a new way; while the examples using $\mathrm{SU}(2)$ and $\mathrm{SU}(3)$ show how in quite elementary situations these conditions may not be obeyed.

Our methods can be and are being applied to study the general structure of phase space formulations of quantum systems and state reconstruction problems.

\section{Acknowledgement}

This work has been done in collaboration with Arvind, S Chaturvedi and R Simon. 


\section{References}

[1] J R Klauder, J. Math. Phys. 4, 1058 (1963)

[2] A Perelomov, Comm. Math. Phys. 26, 22 (1972)

[3] For general information regarding coherent state systems, see, for instance: J R Klauder and E C G Sudarshan, 'Fundamentals of quantum optics (W A Benjamin, New York, 1968), and the excellent review and reprint collection: J R Klauder and B S Skagerstam, Coherent States, (World Scientific Publishing Company, Singapore, 1985)

[4] For an elementary discussion see, for instance, T F Jordan, Linear operators for quantum mechanics (Wiley, New York, 1974)

[5] For some more interesting examples, see, for instance, V Bargmann, P Butera, L Girardello and J R Klauder, Rep. Math. Phys. 2, 221 (1971)

[6] All these are discussed in detail in the second of references [3]

[7] A Perelomov, Generalized coherent states and their applications (Springer-Verlag, 1986)

[8] J E Moyal, Proc. Camb. Philos. Soc. 45, 99 (1949)

[9] S M Roy and V Singh, Phys. Rev. D25, 3413 (1982)

[10] Ref. [7], p. 300

[11] A O Barut and L Girardello, Commun. Math. Phys. 21, 41 (1972)

[12] Arvind, S Chaturvedi, N Mukunda and R Simon, Generalised coherent states and the diagonal representation for operators, quant-ph/0002070

[13] G W Mackey, Group representations in Hilbert space (AMS, Providence, R.I., 1963) 